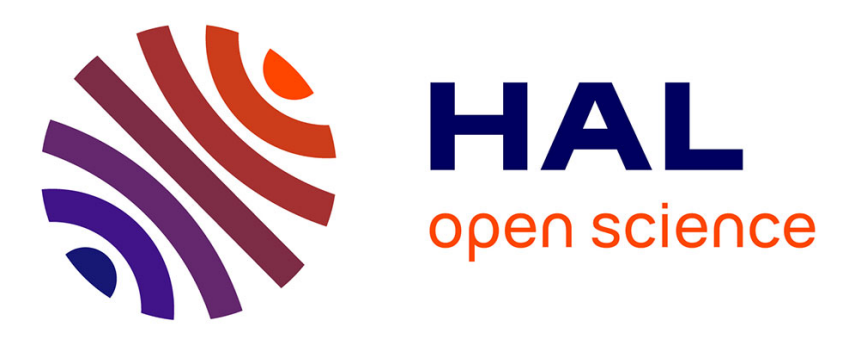

\title{
Modeling of the passive mechanical properties of the musculo-articular complex: Acute effects of cyclic and static stretching
}

Antoine Nordez, Pascal Casari, Christophe Cornu, Jean-Pierre Mariot

\section{- To cite this version:}

Antoine Nordez, Pascal Casari, Christophe Cornu, Jean-Pierre Mariot. Modeling of the passive mechanical properties of the musculo-articular complex: Acute effects of cyclic and static stretching. Innovation and Research in BioMedical engineering, 2009, 30 (1), pp.20-29. 10.1016/j.jbiomech.2008.12.019 . hal-01005847

\section{HAL Id: hal-01005847 https://hal.science/hal-01005847}

Submitted on 20 Nov 2018

HAL is a multi-disciplinary open access archive for the deposit and dissemination of scientific research documents, whether they are published or not. The documents may come from teaching and research institutions in France or abroad, or from public or private research centers.
L'archive ouverte pluridisciplinaire HAL, est destinée au dépôt et à la diffusion de documents scientifiques de niveau recherche, publiés ou non, émanant des établissements d'enseignement et de recherche français ou étrangers, des laboratoires publics ou privés. 


\title{
Modeling of the passive mechanical properties of the musculo-articular complex: Acute effects of cyclic and static stretching
}

\author{
A. Nordez ${ }^{\text {a,* }}$, P. Casari ${ }^{\text {b }}$ J.P. Mariot ${ }^{\text {c }}$, C. Cornu ${ }^{a}$ \\ a Université de Nantes, Laboratoire "Motricité, Interactions, Performance", EA 4334, UFR STAPS, 25 bis bd Guy Mollet BP 72206, Nantes F-44000, France \\ ${ }^{\mathrm{b}}$ Université de Nantes, CNRS, Institut de recherche en Génie Civil et Mécanique, UMR 6183, UFR des Sciences et Techniques, Nantes F-44000, France \\ ' Université du Maine, Laboratoire “Motricité, Interactions, Performance”, EA 4334, UFR STAPS, Avenue Olivier Messiaen, 72085 Le Mans Cedex, France
}

\begin{abstract}
The primary aim of this study was to implement a rheological model of the mechanical behavior of the passive musculo-articular complex (MAC). The second objective was to adapt this model to simulate changes in the passive MAC's mechanical properties induced by passive stretching protocols commonly performed in sport and rehabilitation programs. Nine healthy subjects performed passive ankle dorsiflexion and plantar-flexion cycles at different velocities (from 0.035 to $2.09 \mathrm{rad} \mathrm{s}^{-1}$ ) on an isokinetic dynamometer. This procedure enabled the articular angle to be controlled and the passive torque developed by the MAC in resistance to stretch to be measured. Our rheological model, dependent on nine parameters, was composed of two non-linear (exponential) springs for both plantar- and dorsiflexion, a linear viscoelastic component and a solid friction component. The model was implemented with the Simulink software package, and the nine parameters were identified, for each subject, by minimizing the square-difference between experimental torque-angle relationships and modeled curves. This model is in good agreement with experiment, whatever the considered stretching velocity. Finally, the model was adapted to incorporate static stretching $(4 \times 2.5 \mathrm{~min})$ and cyclic stretching (five loading/unloading cycles) protocols. Our results indicate that the model could be used to simulate the effects of stretching protocols by adjusting a single (different) parameter for each protocol.
\end{abstract}

\section{Introduction}

Passive mechanical properties of a musculo-articular complex (MAC) have been studied experimentally in the last few years in order to characterize acute and chronic changes in range of motion (RoM) (see for review Gajdosik, 2001; Goubel and LenselCorbeil, 2003; Magnusson, 1998; McNair and Portero, 2005). In addition, few studies have modeled some passive mechanical properties of a MAC (Amankwah et al., 2004; Esteki and Mansour, 1996; Riener and Edrich, 1999; Silder et al., 2007). These models have illustrated that passive structures contribute significantly to joint torque during complex movements such as walking (Amankwah et al., 2006; Mansour and Audu, 1986; Whittington et al., 2007). However, these studies take only the elasticity of the MAC into account and, thus ignore the dissipative properties of the MAC, and the velocity dependence of the MAC's response due to its viscosity. Two studies, for example, have shown that energy dissipation via a passive MAC results in about $45 \%$ of the energy being stored during loading (Magnusson et al., 1998; Nordez et al.,

\footnotetext{
* Corresponding author. Tel.: +332518372 38; fax: +33251837210

E-mail address: antoine.nordez@univ-nantes.fr (A. Nordez).
}

2008d). These studies suggest that the dissipative properties of the MAC should be considered when modeling the passive mechanical properties of the MAC.

Indeed, very few studies have modeled both the velocity dependence and the dissipative properties of the passive MAC. Amankwah et al. (2004) have implemented a rheological model that includes velocity dependence of the MAC during loading but which neglects dissipative properties. Similarly, earlier Esteki and Mansour (1996) have described a model in which viscoelastic properties of the metacarpophalangeal joint are treated with quasi-linear viscoelastic theory (Fung, 1983). The power function used to model the relationship between the stretching velocity and the energy dissipation in this case does not agree with recent experimental results for knee joints indicating the existence of a linear (rather than a non-linear) relationship (Nordez et al., 2008b).

In these two last studies (Amankwah et al., 2004; Esteki and Mansour, 1996), the MAC was exercised before the modeling in order to consider the possibility of preconditioning. However, for the MAC in vivo, the preconditioning could be assimilated to a stretching protocol commonly performed in sports or rehabilitation. Indeed, many studies have shown an acute decrease in passive torque and stiffness after cyclic (Magnusson et al., 1998; 
McNair et al., 2001, 2002; Nordez et al., 2008d) and static (Magnusson et al., 1998, 1996; McNair et al., 2001; Nordez et al., 2006) stretching protocols. But to our knowledge, these changes have never been taken into account in a mechanical model of the MAC.

Therefore, the first aim of the present study was to implement and validate a rheological model of the passive ankle MAC's mechanical behavior. Secondly, based on previous experimental observations, the model was adapted to take the acute effects of static and cyclic stretching protocols into account and, hence, to simulate their effects.

\section{Materials and methods}

\subsection{Subjects}

Nine healthy males $(25.6 \pm 2.7$ years, height: $182.0 \pm 8.3 \mathrm{~cm}$, weight: $75.1 \pm 5.7 \mathrm{~kg}$ ) volunteered to participate in this study and signed an informed consent form. This study was conducted according to the Helsinki Statement (1964).

\subsection{Measurement techniques}

The Biodex system 3 research ${ }^{\circledR}$ (Biodex medical, Shirley, NY, USA) isokinetic dynamometer was used in passive mode to perform passive stretching protocols in dorsi-flexion (DF) and plantar-flexion (PF). The dynamometer also measured the ankle angle $(\theta)$, the joint angular velocity $(\omega)$ and the passive torque $(T)$ produced by the ankle joint in resistance to passive motion in reliable and valid ways (Nordez et al., 2008a). Subjects were required to lie on the dynamometer and extend their right leg fully (Nordez et al., 2008c). The input axis of the dynamometer was aligned with the presumed axis of rotation in the right ankle ( 0 rad: foot perpendicular to the leg, positive angles in DF).

To ensure that no undesirable activation occurred during the stretching protocol, bipolar surface electromyographic (sEMG) signals were recorded from surface electrodes (Delsys DE 02.3, Delsys Inc., Boston, USA, $10 \mathrm{~mm}$ inter-electrode distance) and placed on the gastrocnemius lateralis, gastrocnemius medialis and soleus and tibialis anterior muscles in accordance with SENIAM recommendations (Hermens et al., 2000). sEMG signals were visualized in real time by the experimenter and the subject. $T, \theta, \omega$ and SEMG signals were sampled at $1000 \mathrm{~Hz}$ with an analogic/digital converter (Bagnoli 16, Delsys Inc, Boston, USA) and stored in a computer hard disk for further analysis.

\subsection{Experimental protocol (Fig. 1)}

The maximal range of motion (RoM) was first determined in DF (Nordez et al., 2006). The foot was passively dorsi-flexed $\left(0.17 \mathrm{rad} \mathrm{s}^{-1}\right)$ until the maximum tolerable plantar-flexor muscles stretch was reached. This point was operationally defined as the maximal passive DF angle. The best score of three trials was considered as the maximal RoM. Subjects then performed a familiarization with the stretching protocol at the different tested velocities. Subjects were asked to stay as relaxed as possible and they could visualize their sEMG activity using the sEMG feedback. A 10 min rest period was observed between the familiarization and the measurements.

Pre- and post-tests, performed before and after a static stretching protocol $(4 \times 2$ min holds at $50 \%, 60 \%, 70 \%$ and $80 \%$ of the maximal RoM in DF), were identical and composed of

(i) Five passive loading/unloading cycles performed at $0.17 \mathrm{rad} \mathrm{s}^{-1}$ from $0.70 \mathrm{rad}$ in PF until $80 \%$ of the maximal RoM in DF. Previous studies show that no more significant changes occur after the fifth cycle (McNair et al., 2001; Nordez et al., 2008d).

(ii) Five passive loading/unloading cycles performed with the dynamometer preset at $0.035,0.53,1.04,1.57$ and $2.09 \mathrm{rad} \mathrm{s}^{-1}$ in a randomized order (same order for pre- and post-tests) on the same RoM as in (i) (Gajdosik et al., 2005).

Finally, for the sEMG normalization purpose, subjects performed three maximal isometric PF and DF with a 1-min rest period in between. SEMG data was processed according to a previously described procedure (Gajdosik et al., 2005; Nordez et al., 2008b). The sEMG signals were filtered $(6-400 \mathrm{~Hz}$, zero-phase fourth-order Butterworth filter). Root mean squares of the sEMG signals (sEMC RMS) were calculated during stretching and normalized with the maximal level reached during maximal isometric contractions. If the sEMG RMS for a subject was greater than $1 \%$ of the maximum voluntary contraction during stretching trials, then the subject's data were discarded (McNair et al., 2001, 2002). sEMG RMS values were lower than $1 \%$ for all the subjects.

\subsection{Data processing}

All data was processed using a standardized Matlab ${ }^{\mathbb{R}}$ script (The Mathworks, Natick, USA). T, $\theta$ and $\omega$ signals were filtered using a zero-phase low-pass ( $10 \mathrm{~Hz}$ ) fourth-order Butterworth filter and $T$ further corrected for gravity inertia effects. The mass and the inertia of the Biodex level arm were determined during cycles performed at different velocities during an experiment with no subject on the dynamometer (Nordez et al., 2008b). The mass and the inertia of each subjects' foot was determined using regression equations (McFaull and Lamontagne, 1998) provided by Zatsiorski (1998). Using a sensitivity analysis, a previous study demonstrated that inertia estimations predictive equations have negligible influence on passive mechanical properties (Nordez et al., 2008b). The passive torque and angular velocity were determined in increments of $0.007 \mathrm{rad}$ for the 10 cycles of pre- and post-tests.

\subsection{Modeling}

The rheological model used in the present study is composed of four components placed in parallel (Fig. 2): (1) elasticity in PF, (2) elasticity in DF, (3) linear visco-elasticity and (4) solid friction.

The Sten-Knudsen model (Sten-Knudsen, 1953) is known to describe torque-angle relationships accurately and with minimal parameters (Nordez et al., 2006). Therefore, this exponential function was used to model the non-linear elasticity in PF (1) and DF (2)

$T_{1}(\theta)= \begin{cases}\frac{E_{1}}{\alpha_{1}}\left(e^{\alpha_{1}\left(\theta-\theta_{1}\right)}-1\right) & \text { if } \theta \geqslant \theta_{1} \\ 0 & \text { if } \theta<\theta_{1}\end{cases}$

$T_{2}(\theta)= \begin{cases}-\frac{E_{2}}{\alpha_{2}}\left(e^{\alpha_{2}\left(\theta_{2}-\theta\right)}-1\right) & \text { if } \theta \leqslant \theta_{2} \\ 0 & \text { if } \theta>\theta_{2}\end{cases}$

where $T_{1}$ and $T_{2}$ are the passive torques due to tension in the structures on the plantar and dorsal aspects of the ankle, respectively; $E_{i}, \alpha_{i}$ and $\theta_{i}$ are the experimental constants; $\theta_{1}$ and $\theta_{2}$ are the ankle angles at which structures on the plantar and dorsal aspects of the ankle are the slack.

A previous study has revealed a linear relationship between the energy dissipation and the angular velocity (Nordez et al., 2008b), indicating that the dissipative properties of the passive MAC could be modeled using linear viscosity (3) and solid friction (4)

$\dot{T}_{3}=K \dot{\theta}-\frac{K}{\eta} T_{3}$

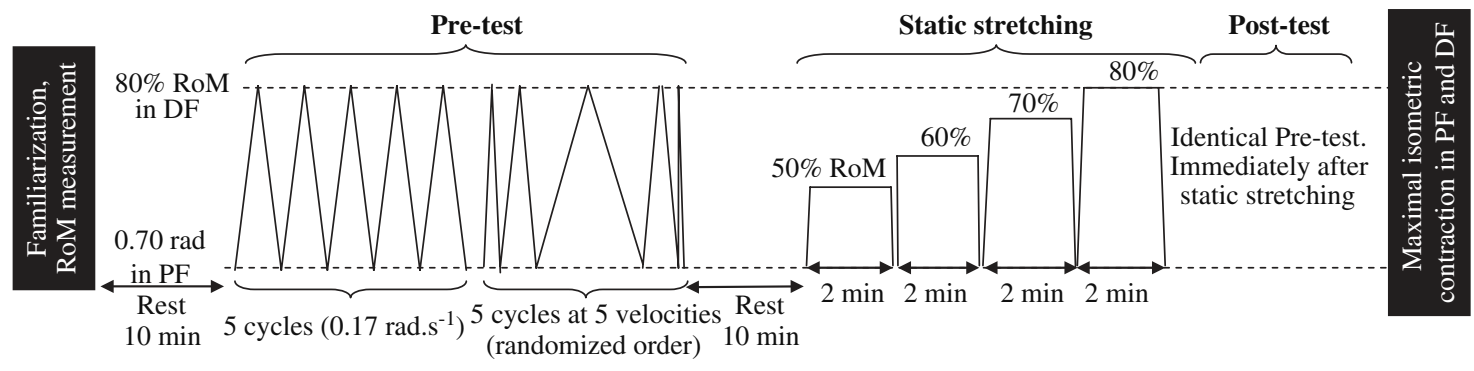

Fig. 1. The experimental protocol. RoM: maximal range of motion, PF: ankle plantar-flexion, DF: ankle dorsi-flexion. 
$T_{4}=f \operatorname{sign}(\dot{\theta}), \quad \operatorname{sign}(x)= \begin{cases}-1 & \text { if } x<0 \\ 1 & \text { if } x>0 \\ 0 & \text { if } x=0\end{cases}$

where $T_{3}$ and $T_{4}$ are the passive torques due to linear viscosity and friction, and $K, \eta$ and $f$ are experimental constants.

The modeled torque is the sum of $T_{1}, T_{2}, T_{3}$ and $T_{4}$. If the stretching velocity is considered as constant, the equations of the rheological model can be solved analytically (Amankwah et al., 2004; Anderson et al., 2002; Wolff et al., 2006).

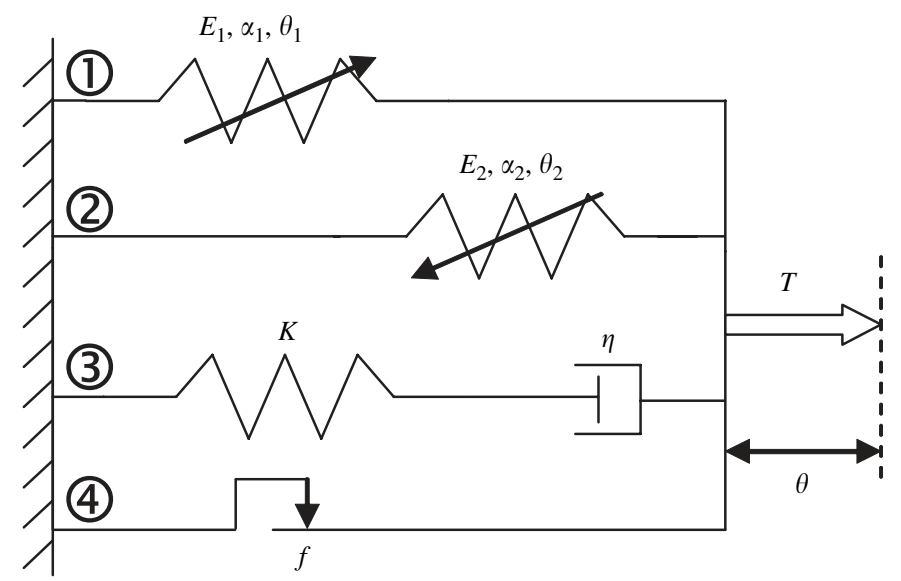

Fig. 2. The rheological model used to simulate the mechanical behavior of the passive musculo-articular complex. It is composed of four components: two nonlinear springs in parallel (1) and (2) (designed to simulate elasticity in dorsi- and plantar-flexion), a linear visco-elasticity factor (3) (in order to simulate velocitydependent energy dissipation) and, finally, a solid friction factor (4) (which simulates velocity-independent energy dissipation).
However, due to the complexity of analytical solutions, the loading history was not taken into account in these previous studies. In addition, a recent study has shown that, because of acceleration capacities of the Biodex system, the angular velocity is not constant for high preset velocities (Nordez et al., 2008a). Therefore, equations of the rheological model were solved numerically using Simulink ${ }^{\mathbb{B}}$ (The Mathworks, Natick, USA) and the ODE45 solver in which 0 was considered the initial condition for $T_{3}$. This approach enabled us to use experimental angle and velocity patterns as inputs for the model. The nine parameters of the model were determined by minimizing the squared error between experimental and modeled data with a non-linear least-squares method (Levenberg-Marquardt algorithm, Simulink Parameter Estimation Toolbox). The parameter calculation was performed using the five cycles performed at five different velocities during the pretest. The parameters were determined using two successive optimizations to fit torque-angle curves (Fig. 3). The six parameters concerning the elasticity in PF (1) and DF (2) were determined using a first optimization to fit torque-angle curves averaged for the loading and unloading, with $T_{3}$ and $T_{4}$ set to 0 . The three remaining parameters concerning the dissipative properties ((3) and (4)) were determined using a second optimization to fit the hysteresis and the velocity dependency with constant values for $E_{i}, \alpha_{i}$ and $\theta_{i}$.

\subsection{Effects of cyclic stretching}

The effects of cyclic stretching were determined using the five cycles performed at $0.17 \mathrm{rad} \mathrm{s}^{-1}$ during the pre-test. Previous work has shown that, during cyclic stretching, the passive torque decreases significantly during loading and only marginally during unloading (Nordez et al., 2008d). Furthermore, a significant decrease in the normalized area of the hysteresis was shown (Magnusson et al., 1998; Nordez et al., 2008d), and these changes did not depend on the stretching velocity (Nordez et al., 2009). Therefore, the effects of cyclic stretching were modeled in this research by an additional solid friction $\left(f_{1}\right)$ factor introduced during the loading phase between the first and fifth cycle

$T_{5}=f_{1}($ cycle $) g(\dot{\theta}), \quad g(x)= \begin{cases}0 & \text { if } x \leqslant 0 \\ 1 & \text { if } x>0\end{cases}$

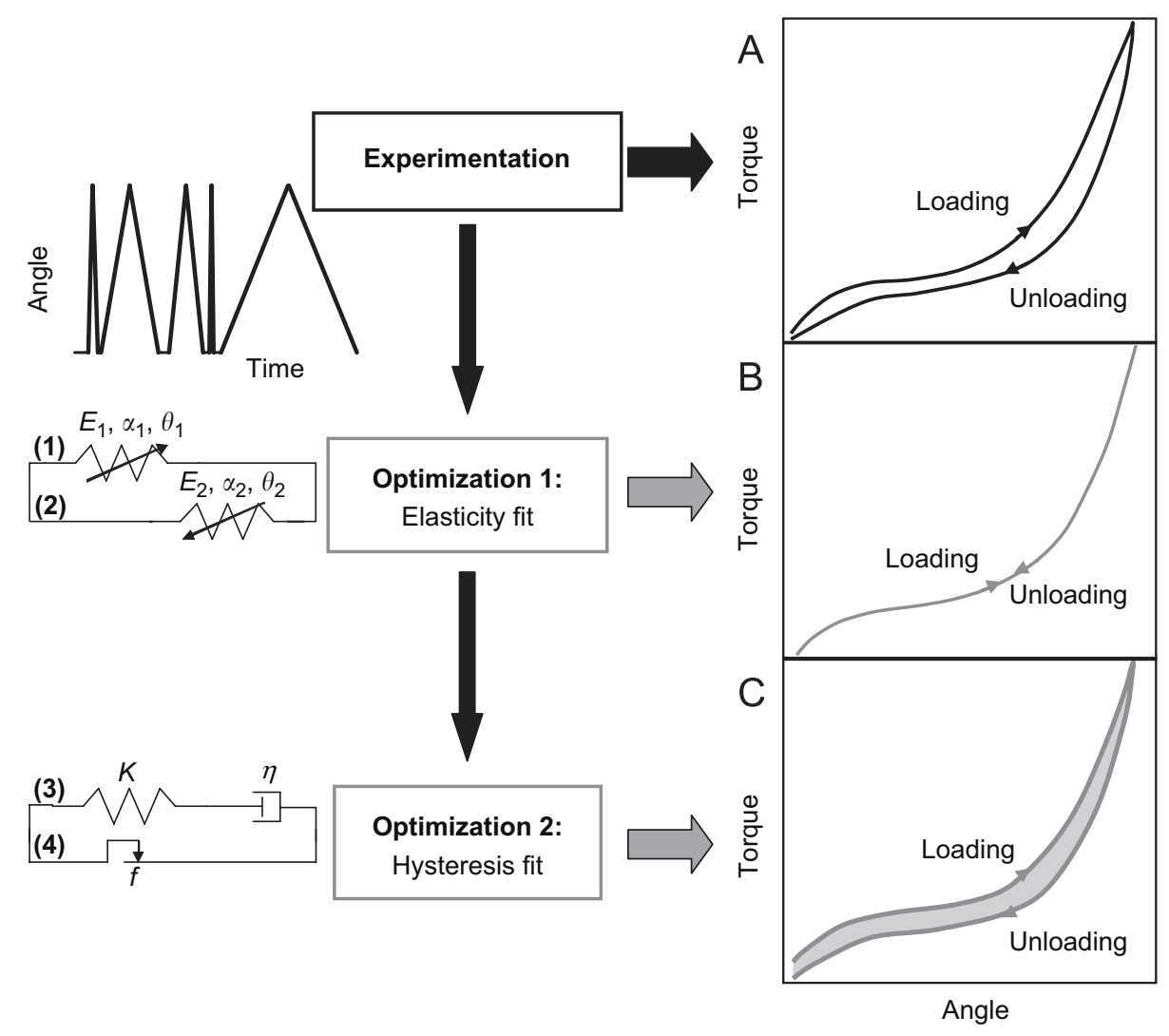

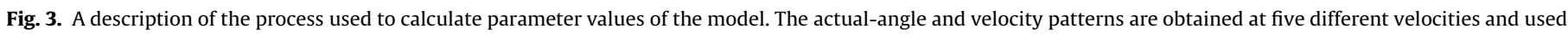

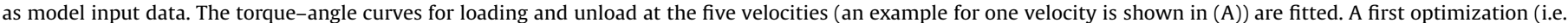

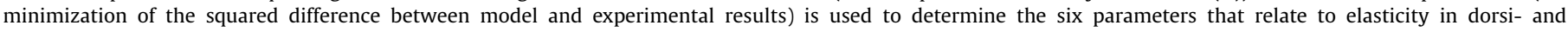

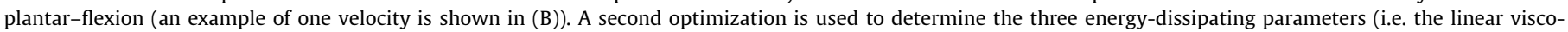
elasticity and the friction) so that hysteresis and velocity dependency can be established (an example of a velocity is shown in (C)). 
Table 1

Parameter $\left(E_{1}, \alpha_{1}, \theta_{1}, E_{2}, \alpha_{2}, \theta_{2}, K, \eta, f\right)$ values of the model (Fig. 2 ) obtained for the nine subjects.

\begin{tabular}{|c|c|c|c|c|c|c|c|c|c|c|}
\hline Subjects & $E_{1}\left(\mathrm{~N} \mathrm{~m} \mathrm{rad}^{-1}\right)$ & $\alpha_{1}\left(\operatorname{rad}^{-1}\right)$ & $\theta_{1}(\mathrm{rad})$ & $E_{2}\left(\mathrm{~N} \mathrm{~m} \mathrm{rad}^{-1}\right)$ & $\alpha_{2}\left(\operatorname{rad}^{-1}\right)$ & $\theta_{2}(\mathrm{rad})$ & $K\left(\mathrm{~N} \mathrm{~m} \mathrm{rad}^{-1}\right)$ & $\eta\left(\mathrm{N} \mathrm{ms} \mathrm{rad}^{-1}\right)$ & $f(\mathrm{~N} \mathrm{~m})$ & RMSE (N m) \\
\hline 1 & 3.78 & 4.42 & -0.258 & 0.460 & 2.94 & 0.648 & 5.24 & 0.748 & 1.95 & 1.28 \\
\hline 2 & 9.37 & 3.57 & -0.127 & 0.322 & 3.40 & 0.547 & 5.31 & 0.718 & 1.63 & 1.20 \\
\hline 3 & 9.08 & 4.43 & -0.185 & 0.230 & 4.91 & 0.468 & 6.71 & 0.745 & 2.20 & 1.42 \\
\hline 4 & 6.16 & 3.97 & -0.237 & 0.517 & 3.73 & 0.365 & 2.74 & 0.774 & 1.16 & 0.80 \\
\hline 5 & 8.80 & 4.31 & -0.169 & 0.422 & 3.62 & 0.527 & 5.57 & 0.976 & 1.83 & 1.29 \\
\hline 6 & 9.42 & 4.24 & -0.055 & 0.368 & 3.48 & 0.668 & 2.71 & 0.983 & 2.38 & 1.53 \\
\hline 7 & 9.72 & 4.29 & -0.158 & 0.506 & 4.35 & 0.419 & 5.22 & 1.022 & 2.11 & 1.30 \\
\hline 8 & 3.63 & 4.07 & -0.309 & 0.733 & 2.93 & 0.200 & 3.94 & 1.109 & 1.18 & 1.34 \\
\hline 9 & 5.61 & 4.36 & -0.266 & 0.459 & 3.40 & 0.506 & 7.42 & 1.590 & 1.63 & 1.89 \\
\hline Mean & 7.29 & 4.18 & -0.196 & 0.446 & 3.64 & 0.483 & 4.98 & 0.963 & 1.79 & 1.34 \\
\hline SD & 2.50 & 0.28 & 0.079 & 0.141 & 0.64 & 0.144 & 1.61 & 0.276 & 0.43 & 0.29 \\
\hline
\end{tabular}

RMSE: root-mean-square error between experimental and modeled torque-angle relationships.

SD: standard deviation.

$f_{1}$ was set to zero during the five cycles at different velocities (after cyclic stretching), and parameter values of the model were set to the values obtained during the modeling step previously described. $f_{1}$ was calculated using a leastsquares numerical minimization of model and experimental data (the Levenberg-Marquardt algorithm).

\subsection{Effects of static stretching}

It has previously been shown that, following static stretching, passive torque is significantly decreased during the loading and the unloading (Nordez et al., 2008d). Furthermore, other studies have shown that no significant changes in the normalized area of the hysteresis exist (Nordez et al., 2008d). Indeed, further observations have indicated that changes in passive torque can be modeled by a "shift to the right" of the passive torque-angle relationship (Nordez, McNair, Casari, Cornu, unpublished observations). Therefore, during the 5th cycle performed during the post-test, we set parameter values to the values obtained during the modeling step previously described, except for $\theta_{1}$, which was estimated using a numerical, least-squares minimization of modeled and experimental data (the Levenberg-Marquardt algorithm).

\subsection{Comparison between experimental and modeled data}

The root mean square error (RMSE) was used to compare modeled and experimental torque-angle relationships. Thereafter, the energy stored during loading ( $E$, i.e. the area under load curve) and the energy dissipated by the musculo-articular complex (ED, i.e. the hysteresis area) were calculated for modeled and experimental data. ED was divided by $E$ to calculate the dissipation coefficient (DC, Nordez et al., 2008d).

After checking the distribution of data (Kolmogorov-Smirnov test), parametric statistical tests were performed using Statistica ${ }^{\mathbb{R}}$ software to analyze the effects of cyclic and static stretching on experimental and modeled results. Four repeated measures analyses of variance (ANOVAs) were used to determine changes in $E$ and DC during the five cycles. An LSD post-hoc analysis was used when appropriate. Four paired $T$-tests were used to assess changes in $E$ and DC between the 5 th cycle of pre- and post-tests. The critical level of significance was set at $P<0.05$.

\section{Results}

\subsection{Modeling}

The parameters obtained for each subject are shown in Table 1. Modeled torque-angle curves were in accordance with experimental results (Fig. 4A and B), and the RMSE between experimental and modeled data was $1.34 \pm 0.29 \mathrm{Nm}$. The energy dissipation was slightly overestimated by our model at the beginning of the RoM, while it was slightly underestimated at the end. However, experimental relationships between $E$, DC and the stretching velocity were still fitted well by our model (Fig. 4C and D).

\subsection{Effects of cyclic stretching}

Between the 1 st and the 5 th cycle $f_{1}$ was decreased by $2.03 \pm 0.52 \mathrm{Nm}$ and the corresponding modeled changes in passive torque-angle curves (Fig. 5B) were similar to experiment (Fig. 5A). The passive torque decreased by a nearly constant value during the loading, but remained relatively unchanged during unloading. In addition, significant decreases in $E$ (Fig. 5C) and DC (Fig. 5D) were found to agree favorably with experiment with the main changes being observed between the first and the second cycle.

\subsection{Effects of static stretching}

The shift in angle between pre- and post-test was $0.042 \pm 0.007 \mathrm{rad}$. Changes in passive torque-angle curves after static stretching were similar for experimental (Fig. 6A) and model (Fig. 6B) data. The passive torque was decreased during the loading and the unloading, with the main changes at the end of the RoM. $E$ was significantly $(P<0.001)$ decreased by $13.7 \pm 4.0 \%$ and $15.7 \pm 6.7 \%$ for experimental and modeled data, respectively (Fig. 6C). In addition, the DC was not significantly $(P>0.05)$ different between experimental and model data (Fig. 6D).

\section{Discussion}

The model used to simulate the mechanical behavior of the passive MAC is composed of four analogical items that exist in parallel: two elastic PF and DF components, a linear viscoelastic component and one solid friction component. The model predicts responses in accordance with experimental results (Fig. 4) and, RMS error values reflect this (approximately $1.3 \mathrm{~N} \mathrm{~m}$, range: $0.80-1.89 \mathrm{Nm}$ ). This RMSE is similar to the error reported by a previous study (range: $0.2-2.3 \mathrm{~N} \mathrm{~m}$ ) (Amankwah et al., 2004) but, in this study, lower passive torque values were obtained in this last study indicating higher percentage error. One other study has modeled both elastic and dissipative properties of a passive MAC (Esteki and Mansour, 1996) but RMS errors are not provided in this paper. The work presented here is an improvement on these previous publications (Amankwah et al., 2004; Esteki and Mansour, 1996), because angle patterns performed during loading and unloading are implicitly included in the model. The StenKnudsen function (Goubel and Lensel-Corbeil, 2003; Sten-Knudsen, 1953) fits torque-angle relationships well and requires only 
A
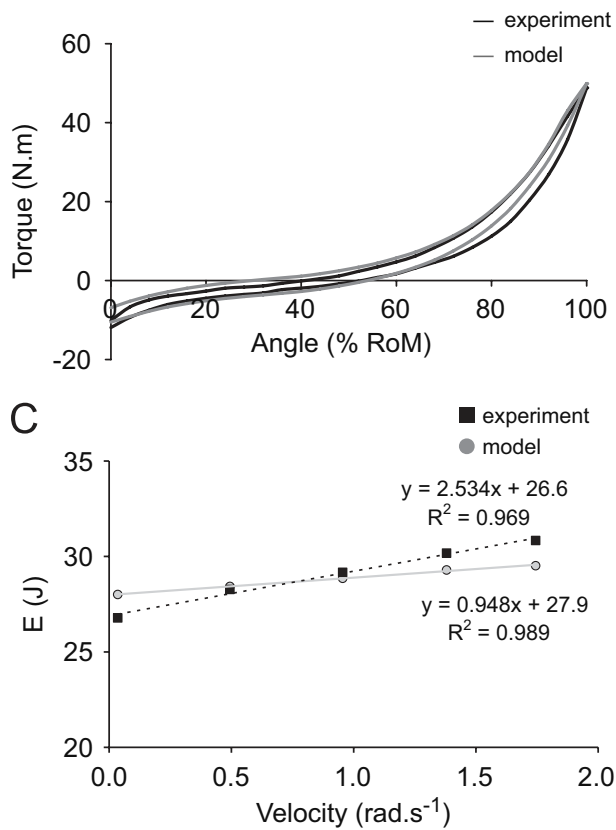

B
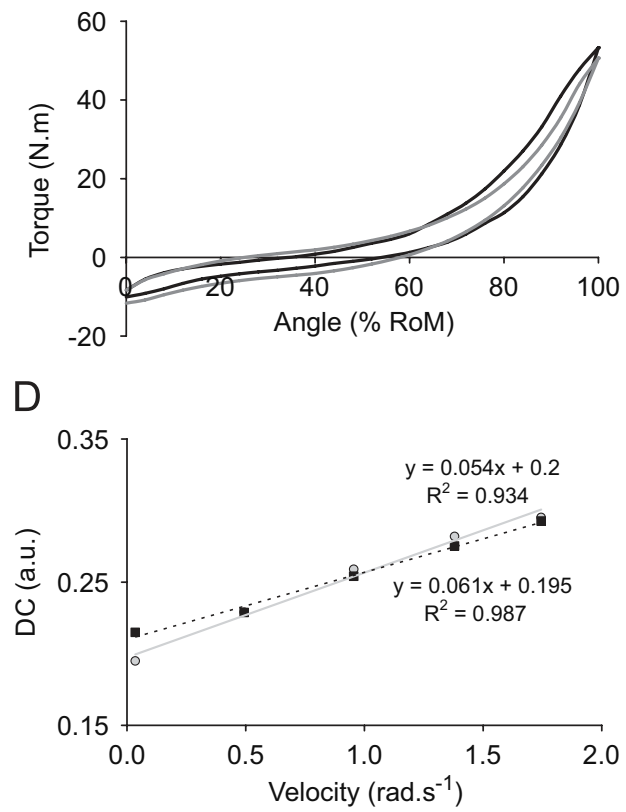

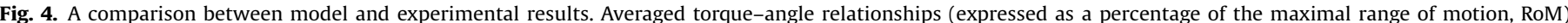

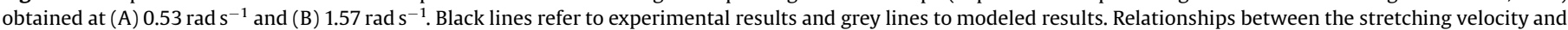
(C) the potential elastic energy stored during the loading (E); (D) the dissipation coefficient (DC). $\mathbf{a}$ experiment, $\bigcirc$ model.

A
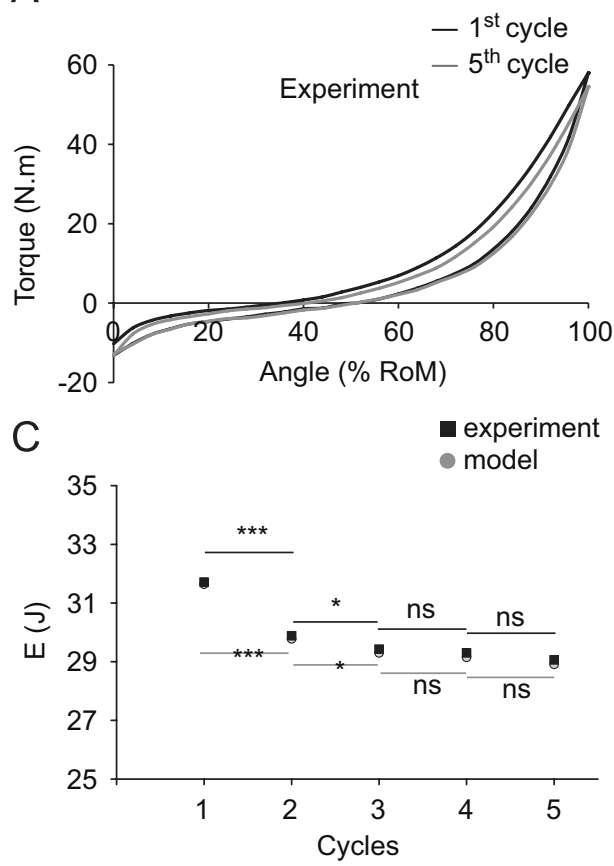

B

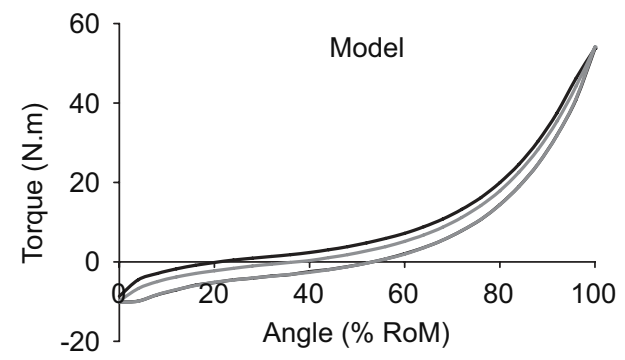

D

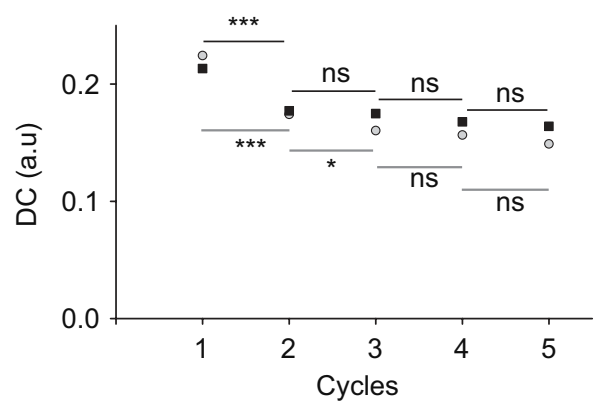

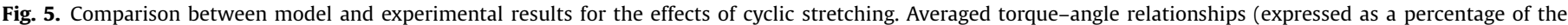

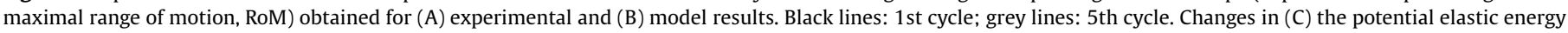
stored during the loading $(E)$ and $(D)$ the dissipation coefficient $(D C)$ during the five cycles. $\mathbf{v}$ experiment, $\bigcirc$ model, ns: non-significant, ${ }^{*} P<0.05$, ${ }^{* * *} P<0.001$.

minimal parameters (Nordez et al., 2006). The exponential function possesses an index, $\alpha$, which represents the gradient of the stiffness-torque function (Nordez et al., 2006). This stiffness index may help to characterize passive stiffness in subject over the whole RoM, using only one parameter.

Our model suffers from some drawbacks. Firstly, our results are only valid in the experimental range of motion ( 0.70 rad in PF to
$80 \%$ of the maximal RoM in DF) and range of stretching velocity $\left(0.035-2.09 \mathrm{rads}^{-1}\right)$. Thus, when the static stretching protocol (Fig. 3) was simulated using our model, the relaxation was underestimated. This underestimation can probably be explained by the superposition of a slow and a fast viscosity as mentioned elsewhere (Esteki and Mansour, 1996; Sauren and Rousseau, 1983; Speich et al., 2006). We have characterized fast viscosity in this 

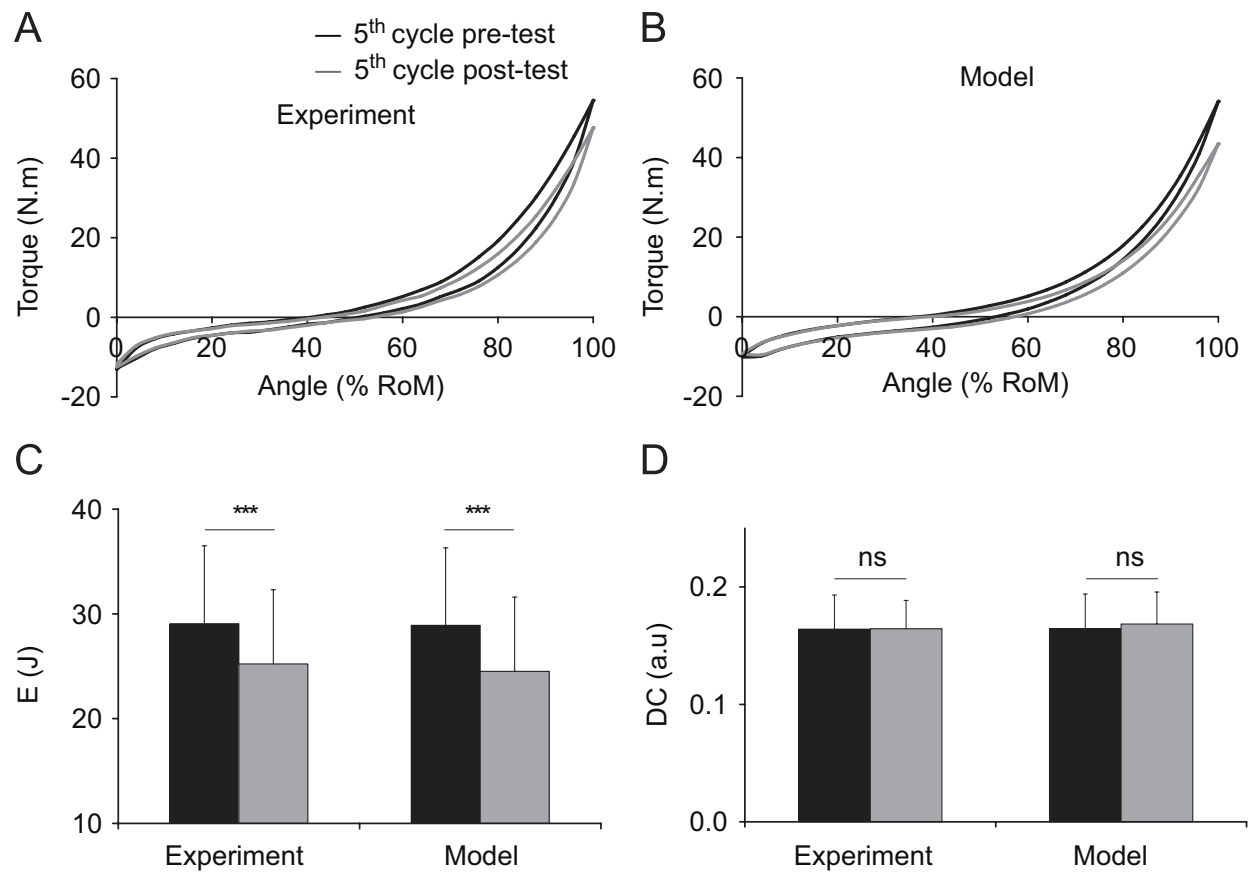

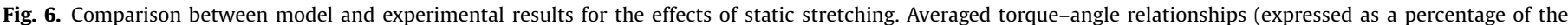

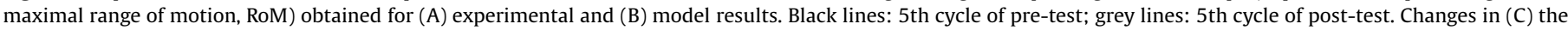

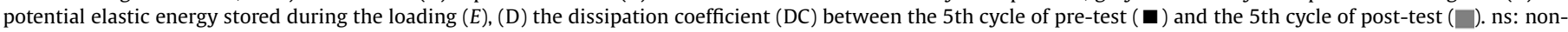
significant, ${ }^{*} \mathrm{P}<0.05,{ }^{* * *} \mathrm{P}<0.001$.

study but further work is needed to assess the slow viscosity using relaxation experiments. Secondly, the model slightly overestimates the dissipation at the beginning of the RoM, while it slightly underestimates the dissipation at the end of the RoM (Fig. 4A and B). Therefore, our model might be improved by including a dissipative factor that increases in response to tissuestretching (Weiss et al., 1986). Because the existence of joint angle dependency increases the number of required parameters; we decided to keep the number of parameters at nine in order to maintain a robust model (Hoang et al., 2005). Improvements will be directed at assessing the joint angle dependency of dissipative properties using more specific experiments (such as sinusoidal perturbations).

Many studies have shown that passive stretching induces an acute decrease in passive torque. Using previous results regarding the effects of cyclic and static stretching (Magnusson et al., 1998; Nordez et al., 2006, 2008d, 2009), our model takes these observations into account and produces good agreement with experiment (Figs. 5 and 6). We conclude that our model can be used to simulate the effects of passive stretching on the passive mechanical properties of the MAC with a satisfactory accuracy. The functional implications of such changes during complex tasks such as walking, running or jumping are not yet well known, and our model could be used to simulate the effects of cyclic and static stretching during these tasks. The main advantage of our model is that elasticity, viscosity and friction are treated separately with passive torque measurements. These model improvements may permit the effects of passive stretching to be understood in a variety of activities (for example, improving sporting performance)

The present study shows that a model that combines two nonlinear elasticities, a linear viscosity and a solid friction enabled us to simulate the mechanical behavior of the passive MAC with a satisfactory accuracy. In addition, based on previous studies, this model was adapted to take into account the effects of cyclic and static stretching. The main advantage of our model is to separate the contributions of elasticity, viscosity and friction in passive torque measurements. Therefore, this model could be used to simulate these contributions in different populations or during complex movements.

\section{Conflict of interest}

All authors disclose any financial and personal relationships with other people or organizations that could inappropriately influence this work.

\section{References}

Amankwah, K., Triolo, R., Kirsch, R., Audu, M., 2006. A model-based study of passive joint properties on muscle effort during static stance. J. Biomech. 39, 2253-2263.

Amankwah, K., Triolo, R.J., Kirsch, R., 2004. Effects of spinal cord injury on lowerlimb passive joint moments revealed througth a nonlinear viscoelastic model. J. Rehabil. Res. Dev. 41, 15-32.

Anderson, J., Li, Z., Goubel, F., 2002. Models of skeletal muscle to explain the increase in passive stiffness in desmin knockout muscle. J. Biomech. 35, 1315-1324.

Esteki, A., Mansour, J.M., 1996. An experimentally based nonlinear viscoelastic model of passive joint moment. J. Biomech. 29, 443-450.

Fung, Y.C., 1983. Biomechanics. Mechanical Properties of Living Tissues. Springer Berlin.

Gajdosik, R.L., 2001. Passive extensibility of skeletal muscle: review of the literature with clinical implications. Clin. Biomech. 16, 87-101.

Gajdosik, R.L., Vander Linden, D.W., McNair, P.J., Riggin, T.J., Albertson, J.S., Mattick, D.J., Wegley, J.C., 2005. Viscoelastic properties of short calf muscle-tendon units of older women: effects of slow and fast passive dorsiflexion stretches in vivo. Eur. J. Appl. Physiol. 95, 131-139.

Goubel, F., Lensel-Corbeil, G., 2003. Biomécanique. Eléments de mécanique musculaire. Masson, Paris.

Hermens, H.J., Freriks, B., Disselhorst-Klug, C., Rau, G., 2000. Development of recommendations for SEMG sensors and sensor placement procedures. J. Electromyogr. Kinesiol. 10, 361-374. 
Hoang, P.D., Gorman, R.B., Todd, G., Gandevia, S.C., Herbert, R.D., 2005. A new method for measuring passive length-tension properties of human gastrocnemius muscle in vivo. J. Biomech. 38, 1333-1341.

Magnusson, S.P., 1998. Passive properties of human skeletal muscle during stretch maneuvers. A review. Scand. J. Med. Sci. Sports 8, 65-77.

Magnusson, S.P., Aagard, P., Simonsen, E., Bojsen-Moller, F., 1998. A biomechanica evaluation of cyclic and static stretch in human skeletal muscle. Int. J. Sports Med. 19, 310-316.

Magnusson, S.P., Simonsen, E.B., Aagaard, P., Kjaer, M., 1996. Biomechanical responses to repeated stretches in human hamstring muscle in vivo. Am. J. Sports Med. 24, 622-628.

Mansour, J.M., Audu, M.L., 1986. The passive elastic moment at the knee and its influence on human gait. J. Biomech. 19, 369-373.

McFaull, S.R., Lamontagne, A., 1998. In vivo measurement of the passive viscoelastic properties of the human knee joint. Hum. Mov. Sci. 17, 139-165.

McNair, P.J., Dombroski, E.W., Hewson, D.J., Stanley, S.N., 2001. Stretching at the ankle joint: viscoelastic responses to holds and continuous passive motion. Med. Sci. Sports Exerc. 33, 354-358.

McNair, P.J., Hewson, D.J., Dombroski, E., Stanley, S.N., 2002. Stiffness and passive peak force changes at the ankle joint: the effect of different joint angular velocities. Clin. Biomech. (Bristol, Avon) 17, 536-540.

McNair, P.J., Portero, P., 2005. Using isokinetic dynamometers for measurements associated with tissue extensibility. Isokinetics Exerc. Sci. 13, 53-56.

Nordez, A., Casari, P., Cornu, C., 2008a. Accuracy of Biodex system 3 pro isokinetic dynamometer in passive mode. Med. Eng. Phys. 30, 880-887.

Nordez, A., Casari, P., Cornu, C., 2008b. Effects of stretching velocity on passive resistance developed by the knee musculo-articular complex: contributions of frictional and viscoelastic behaviours. Eur. J. Appl. Physiol. 103, 243-250.

Nordez, A., Cornu, C., McNair, P., 2006. Acute effects of static stretching on passive stiffness of the hamstring muscles calculated using different mathematical models. Clin. Biomech. 21, 755-760.
Nordez, A., Gennisson, J.L., Casari, P., Catheline, S., Cornu, C., 2008c. Characterization of muscle belly elastic properties during passive stretching using transient elastography. J. Biomech. 41, 2305-2311.

Nordez, A., McNair, P.J., Casari, P., Cornu, C., 2008d. Acute changes in hamstrings musculo-articular dissipative properties induced by cyclic and static stretching. Int. J. Sports Med. 29, 414-418.

Nordez, A., McNair, P.J., Casari, P., Cornu, C., 2009. The effect of angular velocity and cycle on the dissipative properties of the knee during passive cyclic stretching: a matter of viscosity or solid friction. Clin. Biomech. 24, 77-79.

Riener, R., Edrich, T., 1999. Identification of passive elastic joint moments in the lower extremities. J. Biomech. 32, 539-544.

Sauren, A.A., Rousseau, E.P., 1983. A concise sensitivity analysis of the quasi-linear viscoelastic model proposed by Fung. J. Biomech. Eng. 105, 92-95.

Silder, A., Whittington, B., Heiderscheit, B., Thelen, D.G., 2007. Identification of passive elastic joint moment-angle relationships in the lower extremity. J. Biomech. 40, 2628-2635.

Speich, J.E., Quintero, K., Dosier, C., Borgsmiller, L., Koo, H.P., Ratz, P.H., 2006. A mechanical model for adjustable passive stiffness in rabbit detrusor. J. Appl. Physiol. 101, 1189-1198.

Sten-Knudsen, O., 1953. Torsional elsticity of the isolated cross-striated muscle fibre. Acta Physiol. Scand. 28, 1-240.

Weiss, P.L., Kearney, R.E., Hunter, I.W., 1986. Position dependence of ankle joint dynamics-II. Active mechanics. J. Biomech. 19, 737-751.

Whittington, B., Silder, A., Heiderscheit, B., Thelen, D.G., 2007. The contribution of passive-elastic mechanisms to lower extremity joint kinetics during human walking. Gait Posture.

Wolff, A.V., Niday, A.K., Voelker, K.A., Call, J.A., Evans, N.P., Granata, K.P., Grange, R.W., 2006. Passive mechanical properties of maturing extensor digitorum longus are not affected by lack of dystrophin. Muscle Nerve 34, 304-312.

Zatsiorski, V., 1998. Kinematics of Human Motion. Human Kinetics, Champaign, IL. 